\title{
On an Axiomatic about Functional Means
}

\author{
Mustapha Raïssouli $^{1,2}$, Mohamed Chergui ${ }^{3, *}$ and Abdellah Alla ${ }^{4}$ \\ ${ }^{1}$ Department of Mathematics, Science Faculty, Taibah University, Al Madinah Al \\ Munawwarah, P.O.Box 30097, Zip Code 41477, Saudi Arabia \\ 2 Department of Mathematics, Science Faculty, Moulay Ismail University, Meknes, \\ Morocco \\ ${ }^{3}$ Department of Mathematics, LaREAMA Lab, Centre Régional des Métiers de l'Education \\ et de la Formation (CRMEF), Kenitra, Morocco \\ ${ }^{4}$ Department of Mathematics, Mohammed V University, Faculty of Sciences, \\ LAMA-ANLIMAD, Rabat, Morocco
}

Received 7 November 2016; Accepted (in revised version) 7 September 2017

Abstract. In this paper, we introduce an axiomatic approach about functional means. This includes that of operator means already introduced in the literature.

Key Words: Functional mean, operator mean, convex function.

AMS Subject Classifications: 46N10

\section{Introduction}

For over the last centuries, the mean-theory has been the subject of intensive research. Scalar and operator means arise in various contexts and have multiple applications in theoretical point of view as well as in practical purposes.

In recent few years, extension of operator means from the case that the variables are positive linear operators to the case that the variables are convex functionals has been investigated by many authors. Such extensions were introduced in the sense that if $m(T, S)$ is an operator mean between two positive linear operator $T$ and $S$ then its extension $\mathcal{F}(f, g)$ for two functional variables $f$ and $g$ satisfies the following connection-relationship

$$
\mathcal{F}\left(f_{T}, f_{S}\right)=f_{m(T, S)} .
$$

Here the notation $f_{T}$ refers to the convex quadratic function generated by the positive linear operator $T$ acting on a Hilbert space $H$ i.e., $f_{T}(x)=(1 / 2)\langle T x, x\rangle$ for all $x \in H$.

\footnotetext{
*Corresponding author. Email addresses: raissouli .mustapha@gmail .com (M. Raïssouli), chergui_m@yahoo.fr (M. Chergui), abdellah.alla@gmail.com (A. Alla) 
In the aim to recall some standard examples of functional means, we need some notation. Let $H$ be a complex Hilbert space and $f: H \longrightarrow \mathbb{R} \cup\{-\infty, \infty\}$ be a (convex) functional. We denote by $f^{*}$ the conjugate of $f$ defined through

$$
\forall x^{*} \in H, \quad f^{*}\left(x^{*}\right):=\sup _{x \in H}\left(\operatorname{Re}\left\langle x^{*}, x\right\rangle-f(x)\right) .
$$

The arithmetic and harmonic functional means of $f, g: H \longrightarrow \mathbb{R} \cup\{\infty\}$ were introduced in $[1,9,10]$ as follows

$$
\mathcal{A}(f, g):=\frac{f+g}{2}, \quad \mathcal{H}(f, g):=\left(\frac{f^{*}+g^{*}}{2}\right)^{*}=\left(\mathcal{A}\left(f^{*}, g^{*}\right)\right)^{*} .
$$

The geometric functional mean of $f$ and $g$, denoted here by $\mathcal{G}(f, g)$, was firstly introduced in [1] as the point-wise limit of an iterative process descending from the arithmetic and harmonic functional means. Since then some papers were written about $\mathcal{G}(f, g)$ in other points of view, see $[4,8-10]$. Throughout this paper, we adopt as definition of $\mathcal{G}(f, g)$ the following one, see [9] for instance:

$$
\mathcal{G}(f, g)=\frac{1}{\pi} \int_{0}^{1} \frac{1}{\sqrt{t(1-t)}}\left((1-t) f^{*}+t g^{*}\right)^{*} d t
$$

which, under convenient assumptions on $f$ and $g$, is equivalent to that introduced in [1].

The above three functional means satisfy the next double inequality, see $[9,10]$

$$
\mathcal{H}(f, g) \leq \mathcal{G}(f, g) \leq \mathcal{A}(f, g) .
$$

The logarithmic functional mean of $f$ and $g$ was defined by the author in [10] via the following relation:

$$
\mathcal{L}(f, g):=\left(\int_{0}^{1}((1-t) f+t g)^{*} d t\right)^{*} .
$$

The logarithmic functional mean $\mathcal{L}(f, g)$ interpolates $\mathcal{H}(f, g)$ and $\mathcal{A}(f, g)$ in the sense that, see [10]

$$
\mathcal{H}(f, g) \leq \mathcal{L}(f, g) \leq \mathcal{A}(f, g) .
$$

As far as we know, there is no inequality stated in the literature about comparison between $\mathcal{G}(f, g)$ and $\mathcal{L}(f, g)$.

For further details about the above functional means, and more other functional mean$s$, we indicate the references [4,8-10]. We also refer the reader to [6] for some interesting discussion about the geometric functional mean as well as the construction of a parameterized algorithm extending that of $\mathcal{G}(f, g)$.

The fundamental goal of the present paper is to introduce a general definition for means involving (convex) functional arguments $f$ and $g$. In the case where $f$ and $g$ are both quadratic functions generated by positive operators, we immediately obtain in a simple way the operator version of the present functional approach. 


\section{Basic notions and some results}

We first recall some basic notions about convex analysis that will be needed later. For more details, we refer the reader to $[2,3,7,12]$ for instance.

Let $H$ be a complex Hilbert space and $f: H \rightarrow \overline{\mathbb{R}}=\mathbb{R} \cup\{-\infty, \infty\}$ be a functional (in short, we write $f \in \overline{\mathbb{R}}^{H}$ ). The (extended) space $\overline{\mathbb{R}}^{H}$ of such functionals is equipped with the following partial order (so-called the point-wise order)

$$
f, g \in \overline{\mathbb{R}}^{H}, \quad f \leq g \Longleftrightarrow f(x) \leq g(x) \text { for all } x \in H,
$$

where we extend the structure of $\mathbb{R}$ to $\mathbb{R} \cup\{-\infty, \infty\}$ by setting

$$
\forall s \in \mathbb{R} \cup\{-\infty, \infty\}, \quad-\infty \leq s \leq \infty \quad \text { and } \quad s+\infty=\infty .
$$

Since our involved functionals can take infinite values then the equality $f-f=0$ does not always hold, since $\infty-\infty=\infty$. For the same reason, the two inequalities $f \leq g$ and $f-g \leq 0$ are not always equivalent.

We say that $f$ is proper if $f$ does not take the value $-\infty$ and is not identically equal to the value $\infty$. The effective domain $\operatorname{dom} f$ of $f: H \longrightarrow \widetilde{\mathbb{R}}:=\mathbb{R} \cup\{\infty\}$ is given by $\operatorname{dom} f=$ $\{x \in H, f(x)<\infty\}$ and its conjugate $f^{*}$ is defined through (1.1). As it is well-known, $f^{* *}:=\left(f^{*}\right)^{*} \leq f, f^{*}$ is always convex lower semi-continuous and, $f \leq g$ implies $g^{*} \leq f^{*}$, for all functionals $f, g \in \widetilde{\mathbb{R}}^{H}$. We denote by $\Gamma_{0}(H)$ the cone of all convex lower semicontinuous proper functionals. It is well known that $f \in \Gamma_{0}(H)$ if and only if $f^{* *}=f$.

Let $\mathcal{B}(H)$ be the space of bounded linear operators defined from $H$ into itself. As usual, a self-adjoint operator $T \in \mathcal{B}(H)$ is positive (denoted $T \geq 0$ ) if $\langle T x, x\rangle \geq 0$ for all $x \in H$. The set of all (self-adjoint) positive invertible operators $T \in \mathcal{B}(H)$ (in short $T>0$ ) will be denoted by $\mathcal{B}^{+*}(H)$. For $T, S \in \mathcal{B}(H)$, we write $T \leq S$ if and only if $T$ and $S$ are self-adjoint and $S-T \geq 0$.

With this, we state the following example which is of interest.

Example 2.1. Let $T, S$ be two positive linear operators of $H$ and $f_{T}, f_{S}$ their generated quadratic functionals, i.e., $f_{T}(x)=(1 / 2)\langle T x, x\rangle$ for every $x \in H$. Then we have:

(i) $f_{T} \pm f_{S}=f_{T \pm S}$ and, $f_{T}=(\geq \leq) f_{S}$ if and only if $T=(\geq \leq) S$.

(ii) $\operatorname{dom} f_{T}=H$ and $f_{T} \in \Gamma_{0}(H)$.

(iii) If moreover $T$ is invertible then $f_{T}^{*}(x)=(1 / 2)\left\langle T^{-1} x, x\right\rangle$ for all $x \in H$. We then write $f_{T}^{*}=f_{T^{-1}}$.

Proposition 2.1. Let $f: H \longrightarrow \mathbb{R} \cup\{\infty\}$. Then there hold:

(i) If $f$ is not identically equal to $\infty$ then $f^{*}>-\infty$ i.e., $f^{*}$ does not take the value $-\infty$.

(ii) If $f$ is convex lower semi-continuous and not identically equal to $\infty$ then so is $f^{*}$. That is, $f \in \Gamma_{0}(H)$ implies $f^{*} \in \Gamma_{0}(H)$. 
Proof. (i) By hypothesis, there is $x_{0} \in H$ such that $f\left(x_{0}\right)<\infty$. Otherwise, from (1.1) we deduce, for every $x^{*} \in H$,

$$
f^{*}\left(x^{*}\right) \geq \operatorname{Re}\left\langle x^{*}, x_{0}\right\rangle-f\left(x_{0}\right)>-\infty .
$$

(ii) In fact $f^{*}$ is always convex lower semi-continuous even $f$ is not. We have only to show that $f^{*}$ is not identically equal to $\infty$. For this, see (see [2], Proposition I.9, page 9) for instance, where the desired result is established by using the geometric version of the celebrate Hahn-Banach theorem.

Before giving another result, we need more notations. We define

$$
\begin{aligned}
& D^{+}(H):=\left\{(f, g) \in \Gamma_{0}(H) \times \Gamma_{0}(H), \operatorname{dom} f \cap \operatorname{dom} g \neq \varnothing\right\}, \\
& D^{+*}(H):=\left\{(f, g) \in \Gamma_{0}(H) \times \Gamma_{0}(H), \operatorname{dom} f^{*} \cap \operatorname{dom} g^{*} \neq \varnothing\right\} .
\end{aligned}
$$

Obviously, $D^{+}(H)$ and $D^{+*}(H)$ are two nonempty sub-cones of the product-cone $\Gamma_{0}(H) \times$ $\Gamma_{0}(H)$, with the next implications

$$
(f, g) \in D^{+}(H) \Longrightarrow\left(f^{*}, g^{*}\right) \in D^{+*}(H) \Longrightarrow \operatorname{dom} f \cap \operatorname{dom} g \neq \varnothing .
$$

Further, it is easy to see that $\left(f_{T}, f_{S}\right) \in D^{+}(H) \cap D^{+*}(H)$ for all $T, S \in \mathcal{B}^{+*}(H)$.

We now may state the following result.

Proposition 2.2. (i) Let $f, g \in D^{+}(H)$. Then $\mathcal{A}(f, g) \in \Gamma_{0}(H)$.

(ii) Let $f, g \in D^{+*}(H)$. Then $\mathcal{H}(f, g) \in \Gamma_{0}(H), \mathcal{G}(f, g) \in \Gamma_{0}(H)$ and $\mathcal{L}(f, g) \in \Gamma_{0}(H)$.

Proof. (i) Assume that $f, g \in D^{+}(H)$. It is clear that $\mathcal{A}(f, g)$ is convex, lower semicontinuous and $\mathcal{A}(f, g)>-\infty$. Since $\operatorname{dom} f \cap \operatorname{dom} g \neq \varnothing$ then there is $x_{0} \in H$ such that $f\left(x_{0}\right)<\infty$ and $g\left(x_{0}\right)<\infty$. We then deduce $\mathcal{A}(f, g)\left(x_{0}\right)<\infty$ and the desired result follows.

(ii) By the previous proposition, we have $f^{*}, g^{*} \in \Gamma_{0}(H)$ and by the above (i) we obtain $\mathcal{A}\left(f^{*}, g^{*}\right) \in \Gamma_{0}(H)$. Again, by the same above proposition we deduce that $\mathcal{H}(f, g):=$ $\left(\mathcal{A}\left(f^{*}, g^{*}\right)\right)^{*} \in \Gamma_{0}(H)$.

The fact that $\mathcal{G}(f, g) \in \Gamma_{0}(H)$ and $\mathcal{L}(f, g) \in \Gamma_{0}(H)$ follow immediately from (1.4) and (1.6), respectively. The proof is so complete.

Proposition 2.3. Let $f, g, h, k: H \longrightarrow \mathbb{R} \cup\{\infty\}$ be four given functionals. Then we have:

$$
\mathcal{A}(\mathcal{A}(f, g), \mathcal{A}(f, h))=\mathcal{A}(f, \mathcal{A}(g, h)), \quad \mathcal{A}(\mathcal{A}(f, g), \mathcal{A}(h, k))=\mathcal{A}(\mathcal{A}(f, h), \mathcal{A}(g, k)) .
$$

Furthermore,

$$
\mathcal{H}(\mathcal{H}(f, g), \mathcal{H}(f, h))=\mathcal{H}(f, \mathcal{H}(g, h)),
$$

provided that $(f, g),(f, h),(g, h) \in D^{+*}(H)$, and

$$
\mathcal{H}(\mathcal{H}(f, g), \mathcal{H}(h, k))=\mathcal{H}(\mathcal{H}(f, h), \mathcal{H}(g, k)),
$$

provided that $(f, g),(h, k),(f, h),(g, k) \in D^{+*}(H)$. 
Proof. The proof of the first equality and that of the second one are simple and comes from the fact that $\mathcal{A}(f, g)$ has a linear affine character with respect to its variables $f$ and g. We will prove the third equality. Indeed, by definition of $\mathcal{H}$ we have

$$
\mathcal{H}(\mathcal{H}(f, g), \mathcal{H}(f, h))=\mathcal{H}\left(\left(\mathcal{A}\left(f^{*}, g^{*}\right)\right)^{*},\left(\mathcal{A}\left(f^{*}, h^{*}\right)\right)^{*}\right) .
$$

Since $f, g \in \Gamma_{0}(H)$ then $f^{*}, g^{*} \in \Gamma_{0}(H)$, too. According to the previous we then deduce $\mathcal{A}\left(f^{*}, g^{*}\right) \in \Gamma_{0}(H)$. Similarly, we prove that $\mathcal{A}\left(f^{*}, h^{*}\right) \in \Gamma_{0}(H)$. It follows that

$$
\left(\mathcal{A}\left(f^{*}, g^{*}\right)\right)^{* *}=\mathcal{A}\left(f^{*}, g^{*}\right) \text { and }\left(\mathcal{A}\left(f^{*}, h^{*}\right)\right)^{* *}=\mathcal{A}\left(f^{*}, h^{*}\right) .
$$

With this, we can write

$$
\mathcal{H}(\mathcal{H}(f, g), \mathcal{H}(f, h))=\left(\mathcal{A}\left(\mathcal{A}\left(f^{*}, g^{*}\right), \mathcal{A}\left(f^{*}, h^{*}\right)\right)\right)^{*},
$$

which, with the above first equality, yields

$$
\mathcal{H}(\mathcal{H}(f, g), \mathcal{H}(f, h))=\left(\mathcal{A}\left(f^{*}, \mathcal{A}\left(g^{*}, h^{*}\right)\right)\right)^{*}=\mathcal{H}\left(f,\left(\mathcal{A}\left(g^{*}, h^{*}\right)\right)^{*}\right)=\mathcal{H}(f, \mathcal{H}(g, h)) .
$$

The fourth equality can be proved in a similar manner as the third one.

Before stating another result, we introduce more notions as recited in the following:

Definition 2.1. Let $\left(f_{n}\right)$ be a sequence of functionals defined from $H$ into $\mathbb{R} \cup\{\infty\}$ :

(i) We say that $\left(f_{n}\right)$ is point-wisely increasing (in short $p$-increasing) if for all $n, f_{n} \leq f_{n+1}$ for the point-wise order.

(ii) We say that $\left(f_{n}\right)$ is point-wisely convergent (in short $p$-convergent) if there is a functional $f: H \longrightarrow \mathbb{R} \cup\{-\infty, \infty\}$ such that $\lim _{n \uparrow \infty} f_{n}(x)=f(x)$ for all $x \in H$.

The following example, which illustrates the above definition, is of interest.

Example 2.2. Let $f_{n}(x)=(1 / 2)\left\langle T_{n} x, x\right\rangle$ for all $x \in H$, where $\left(T_{n}\right)$ is a sequence of selfadjoint linear operators. Then:

(i) $\left(f_{n}\right)$ is $p$-increasing (resp. $p$-decreasing) if and only if $\left(T_{n}\right)$ is increasing (resp. decreasing) for the Loẅner order.

(ii) $\left(f_{n}\right) p$-converges if and only if for all $x \in H, \lim _{n \uparrow \infty}\left\langle T_{n} x, x\right\rangle=f(x)$ for some functional $f$. If moreover, $f$ is with finite values then there exists a self-adjoint linear operator $T$ such that $f=f_{T}$. With this, $\left(f_{n}\right) p$-converges to $f$ if and only if $\left(T_{n}\right)$ converges quadratically to $T$. Further, it is well-known that, if $T_{n} \geq T$ then, $\left(T_{n}\right)$ converges quadratically to $T$ if and only if $\left(T_{n}\right)$ converges strongly (i.e., in norm) to $T$.

Now, we are in position to state our desired result recited as follows. 
Proposition 2.4. Let $\left(f_{n}\right)$ be a $p$-decreasing sequence of functionals $p$-converging to $f$. Then $\left(f_{n}^{*}\right) p$-converges increasingly to $f^{*}$.

Proof. First, remark that $\left(f_{n}^{*}\right)$ is $p$-increasing since $\left(f_{n}\right)$ is $p$-decreasing. The fact that $\left(f_{n}\right)$ $p$-converges decreasingly to $f$ is equivalent to $f=\lim _{n} f_{n}$, where the infimum is taken here for the point-wise order. We wish to establish that $f^{*}=\sup _{n} f_{n}^{*}$. We have, for all $x^{*} \in H$,

$$
\begin{aligned}
\sup _{n} f_{n}^{*}\left(x^{*}\right) & =\operatorname{supsup}_{n}\left\{\operatorname{Re}\left\langle x^{*}, x\right\rangle-f_{n}(x)\right\} \\
& =\operatorname{supsup}_{x \in H}\left\{\operatorname{Re}\left\langle x^{*}, x\right\rangle-f_{n}(x)\right\} \\
& =\sup _{x \in H}\left\{\operatorname{Re}\left\langle x^{*}, x\right\rangle-\inf _{n} f_{n}(x)\right\} \\
& =\sup _{x \in H}\left\{\operatorname{Re}\left\langle x^{*}, x\right\rangle-f(x)\right\} \\
& =f^{*}\left(x^{*}\right),
\end{aligned}
$$

which is the desired result, so completes the proof of the proposition.

\section{Axiomatic theory about functional means}

This section displays an axiomatic study about functional means. We first recall the following, see [11].

Definition 3.1. Let $\mathbf{M}: \mathcal{B}^{+*}(H) \times \mathcal{B}^{+*}(H) \longrightarrow \mathcal{B}^{+*}(H)$ be a binary map satisfying the following list of assertions:

$\left(o_{1}\right) \mathbf{M}(T, T)=T$ for all $T \in \mathcal{B}^{+*}(H)$;

$\left(o_{2}\right) \mathbf{M}(T, S)=\mathbf{M}(S, T)$ for all $T, S \in \mathcal{B}^{+*}(H)$;

$\left(o_{3}\right) \mathbf{M}(\lambda T, \lambda S)=\lambda \mathbf{M}(T, S)$ for all $T, S \in \mathcal{B}^{+*}(H)$ and every real number $\lambda>0$;

$\left(o_{4}\right)$ For all $T_{1}, T_{2}, S_{1}, S_{2} \in \mathcal{B}^{+*}(H)$ such that $T_{1} \leq T_{2}$ and $S_{1} \leq S_{2}$ we have $\mathbf{M}\left(T_{1}, S_{1}\right) \leq$ $\mathbf{M}\left(T_{2}, S_{2}\right)$. In this case, we say that $\mathbf{M}$ is a monotone operator mean.

The standard examples of monotone operator means are the following:

$$
\begin{aligned}
& \mathbf{A}(T, S)=\frac{T+S}{2} \\
& \mathbf{H}(T, S)=\left(A\left(T^{-1}, S^{-1}\right)^{-1}=2 T(T+S)^{-1} S,\right. \\
& \mathbf{G}(T, S)=T^{1 / 2}\left(T^{-1 / 2} S T^{-1 / 2}\right)^{1 / 2} T^{1 / 2}, \\
& \mathbf{L}(T, S)=\left(\int_{0}^{1}((1-t) T+t S)^{-1} d t\right)^{-1},
\end{aligned}
$$


and are known as the arithmetic, harmonic, geometric and logarithmic operator means, respectively. For further examples about monotone operator means (not needed here) we refer the reader to [11] and the related references cited therein. As pointed out in [11], monotone operator means adopted here include those of Kubo-Ando ones, [5]. The above four operator means satisfy the following chain of inequalities

$$
\mathbf{H}(T, S) \leq \mathbf{G}(T, S) \leq \mathbf{L}(T, S) \leq \mathbf{A}(T, S) .
$$

In the scalar case (i.e., $\operatorname{dim} H=1$ ) they are, respectively, reduced to the so-called scalar means, namely

$$
A(a, b)=\frac{a+b}{2}, \quad H(a, b)=\frac{2 a b}{a+b}, \quad G(a, b)=\sqrt{a b}, \quad L(a, b)=\frac{a-b}{\ln a-\ln b} \quad \text { with } L(a, a)=a,
$$

for all real numbers $a>0, b>0$.

Now, we are in position to state our adopted definition about functional means recited as follows.

Definition 3.2. By functional mean we understand a binary map $\mathcal{F}$ between $\Gamma_{0}(H)$ functionals satisfying the following requirements:

$\left(f_{1}\right) \mathcal{F}(f, f)=f$ for all $f \in \Gamma_{0}(H)$;

$\left(f_{2}\right) \mathcal{F}(f, g)=\mathcal{F}(g, f)$ for all $f, g \in \Gamma_{0}(H)$;

$\left(f_{3}\right) \mathcal{F}(t . f, t . g)=t . \mathcal{F}(f, g)$ for all $f, g \in \Gamma_{0}(H)$ and each real number $t>0$;

$\left(f_{4}\right) \mathcal{F}(f, g)$ is point-wisely increasing in $f$ (and in $g$ ), that is for all $f_{1}, f_{2} \in \Gamma_{0}(H)$ such that $f_{1} \leq f_{2}$ and every $g \in \Gamma_{0}(H)$, we have $\mathcal{F}\left(f_{1}, g\right) \leq \mathcal{F}\left(f_{2}, g\right)$;

(of) For all $T, S \in \mathcal{B}^{+*}(H)$, we have

$$
\mathcal{F}\left(f_{T}, f_{S}\right)=f_{\mathbf{M}(T, S)},
$$

where $\mathbf{M}$ is a monotone operator mean. In this case, we say that $\mathcal{F}$ is the $\mathbf{M}$-functional mean.

Obviously, the set of all functional means is a convex cone. Otherwise, it is easy to see that the class of functional means adopted here include that introduced by Fujii in [4].

The standard examples of functionals means known in the literature are $\mathcal{A}(f, g)$, $\mathcal{H}(f, g), \mathcal{G}(f, g)$ and $\mathcal{L}(f, g)$ previously defined. These are known as the arithmetic, harmonic, geometric and logarithmic functional means, respectively, since they satisfy (following the above requirement (of))

$$
\mathcal{A}\left(f_{T}, f_{S}\right)=f_{\mathbf{A}(T, S)}, \quad \mathcal{H}\left(f_{T}, f_{S}\right)=f_{\mathbf{H}(T, S)}, \quad \mathcal{G}\left(f_{T}, f_{S}\right)=f_{\mathbf{G}(T, S)}, \quad \mathcal{L}\left(f_{T}, f_{S}\right)=f_{\mathbf{L}(T, S)},
$$

for all $T, S \in \mathcal{B}^{+*}(H)$, where $\mathbf{A}(T, S), \mathbf{H}(T, S), \mathbf{G}(T, S)$ and $\mathbf{L}(T, S)$ are defined by (3.1a), (3.1b), (3.1c) and (3.1d), respectively.

The following remark is of interest. 
Remark 3.1. The assertions $\left(f_{1}\right)-\left(f_{4}\right)$, for defining a functional mean, were put by analogy with $\left(o_{1}\right)-\left(o_{4}\right)$, respectively. However, the assertion (of), giving a connectionrelationship between functional mean and its related operator mean, is here taken as primordial condition. This is in the aim to have nice properties and interesting examples of functional means. The following counter-example explains more precisely this latter situation.

Example 3.1. Let $f, g \in \Gamma_{0}(H)$ and consider the maps $(f, g) \longmapsto \inf (f, g)$ and $(f, g) \longmapsto$ $\sup (f, g)$, where the infimum and the supremum are taken for the point-wise order. It is easy to see that these two functional-maps satisfy the requirements $\left(f_{1}\right)-\left(f_{4}\right)$. However, they do not satisfy $(o f)$ in general, because the algebra $\mathcal{B}(H)$ is not totaly ordered when $\operatorname{dim} H \geq 2$. In another way, $(f, g) \longmapsto \inf (f, g)$ and $(f, g) \longmapsto \sup (f, g)$ are in general not functional means.

Now, the following result may be stated.

Proposition 3.1. Let $\mathcal{F}$ be a functional mean. Then the following functional-inequalities

$$
(\inf (f, g))^{* *} \leq \mathcal{F}(f, g) \leq \sup (f, g)
$$

hold true for all $(f, g) \in D^{+}(H)$.

Proof. Let $(f, g) \in D^{+}(H)$. It is not hard to see that $(\inf (f, g))^{* *}$ and $\sup (f, g)$ belong to $\Gamma_{0}(H)$. Now, writing

$$
\begin{aligned}
& (\inf (f, g))^{* *} \leq \inf (f, g) \leq f \leq \sup (f, g), \\
& (\inf (f, g))^{* *} \leq \inf (f, g) \leq g \leq \sup (f, g),
\end{aligned}
$$

we then deduce, by $\left(f_{4}\right)$ and $\left(f_{1}\right)$, the desired result after a simple manipulation.

As it is well-known, even if $f, g \in \Gamma_{0}(H)$ are convex, $\inf (f, g)$ is not convex in general, and so $(\inf (f, g))^{* *} \neq \inf (f, g)$ for $f, g \in D^{+}(H)$.

We now end this section by stating the following result.

Proposition 3.2. Let $\mathcal{F}_{n}$ be a sequence of functional means. Assume that, for all $f, g \in$ $\Gamma_{0}(H)$, we have

$$
\lim _{n \uparrow \infty} \mathcal{F}_{n}(f, g)=\mathcal{F}(f, g),
$$

where the limit is taken for the point-wise convergence. Then $\mathcal{F}$ is a functional mean.

Proof. It is easy to verify that, if $\mathcal{F}_{n}$ satisfies $\left(f_{1}\right)-\left(f_{4}\right)$ for each $n$, then so is $\mathcal{F}$. Only the requirement $(o f)$ needs some detail.

So, let us prove that $\mathcal{F}\left(f_{T}, f_{S}\right)=f_{\mathbf{M}(T, S)}$, for some monotone operator mean $\mathbf{M}$. By virtue of our assumption we can write for all integer $n, \mathcal{F}_{n}\left(f_{T}, f_{S}\right)=f_{\mathbf{M}_{n}(T, S)}$ with $\mathbf{M}_{n}$ is a monotone operator mean. Since $\left(\mathcal{F}_{n}\left(f_{T}, f_{S}\right)\right)$ is point-wisely convergent we can then put $\lim f_{\mathbf{M}_{n}(T, S)}=f(T, S)$. Now, according to Example 2.2(ii) we can state $f(T, S)=f_{\mathbf{M}(T, S)}$ and consequently $\mathcal{F}\left(f_{T}, f_{S}\right)=f_{\mathbf{M}(T, S)}$. We now need to show that $\mathbf{M}$ is a monotone operator mean, i.e., $\mathbf{M}$ satisfies the assertions $\left(o_{1}\right)-\left(o_{4}\right)$ of Definition 3.1. This can be checked in an elementary way. We omit the details which are very simple. 


\section{References}

[1] M. Atteia and M. Raïssouli, Self dual operators on convex functionals, geometric mean and square root of convex functionals, J. Convex Anal., 8(1) (2001), 223-240.

[2] H. Brézis, Analyse Fonctionnelle, Theory et Applications, Masson, 1986.

[3] I. Ekeland and R. Temam, Convex Analysis and Variational Problems, SIAM, 1999.

[4] J.I. Fujii, Kubo-Ando theory of convex functional means, Sci. Math. Japon., 7 (2002), 299-311.

[5] F. Kubo and T. Ando, Means of positive linear operators, Math. Ann. 246 (1980), 205-224.

[6] S. Kum and Y. Lim, A geometric mean of parameteized arithmetic and harmonic means of convex functions, Abstract Appl. Anal., (2012), 836804.

[7] P.J. Laurent, Approximation et Optimisation, Hermann, 1972.

[8] M. Raïssouli and M. Chergui, Arithmetico-geometric and geometrico-harmonic means of two convex functionals, Sci. Math. Japonicae, 55(3) (2002), 485-492.

[9] M. Raïssouli and H. Bouziane, Arithmetico-geometrico-harmonic functional mean in convex analysis, Ann. Sci. Math. Quebec, 30(1) (2006), 79-107.

[10] M. Raïssouli, Logarithmic functional mean in convex analysis, J. Ineq. Pure Appl. Math., 10(4) (2009).

[11] M. Raïssouli, Stable and stabilizable means involving linear operator arguments, Linear and Multilinear Algebra, 62(9) (2014), 1153-1168.

[12] E.Zeidler, Nonlinear Functional Analysis and Its Applications III, Springer-Verlag, 1984. 